\title{
On Parabolic Functions of One-Dimensional Quasidiffusions
}

By

\author{
Uwe KÜCHLER*
}

\section{§1. Introduction and Definitions}

In this paper we will deal with quasidiffusions $X=\left(X_{t}\right)_{t \geqq 0}$ on $[0,1)$ assuming that 0 is a reflecting regular boundary, 1 is an accessible or entrance boundary and $X$ is killed as soon as it hits the boundary 1 . We shall give a Martin representation of space-time-excessive functions for $X$. This includes in particular a representation of all parabolic functions $f(x, t)$ satisfying a certain integrability condition by minimal ones (see Theorem 2 below).

We shall show that the set of minimal points of space-time Martin boundary is homeomorphic to $(0, \infty[$; in particular the minimal parabolic functions form a one-parameter family $\left(k_{t}\right)(t \in(0, \infty])$. If $t<\infty$, the function $k_{t}(x, s)$ is the limit (in a weak sense) of the transition density $p(t-s, x, y)$ or its derivative with respect to $y$, where $y$ converges to the boundary 1 . In the limit circle case we will give an uniformly convergent expansion of $k_{t}(t \in(0, \infty])$ in eigenfunctions of the infinitesimal operator of $X$.

Using these results we consider the problem which minimal parabolic functions factorize (i.e. have the form $k_{t}(x, s)=\phi_{t}(x) \psi_{t}(s)$ ) and which factorizing parabolic functions are minimal. (For some Markov chains and diffusion processes this problem was studied in [9], [11].)

As another application we shall give a necessary and sufficient condition in order that for a parabolic function $f$ the process $\left\{f\left(X_{t}, s+t\right), t \in[a, b]\right\}$ is a martingale. Parabolic functions which are martingales on the trajectories of $X$ are used to determine the probabilities that $X$ ever hits some time-varying boundaries (see e.g. [12]) and were studied e.g. in [2], [8].

The assumption that the boundary 1 is accessible or entrance is essential.

Communicated by K. Itô, March 17, 1978.

* Sektion Mathematik, Technische Universität Dresden, Mommsenstrasse 13, DDR-8027 Dresden, German Democratic Republic. 
If 1 is natural then the transition densities and their derivatives have a complete other asymptotic behaviour and therefore the minimal parabolic functions are of other type. This case will be studied in a further note (see [6]).

In the following we will use the terminology and the results of [5] without further explanation.

Let $(m, p)$ be a canonical pair (see [5]) and $X:=\left(X_{t}\right)_{t \geqq 0}$ the corresponding quasidiffusion on $E:=\operatorname{supp} m \backslash\{1\} \subseteq[0,1)$ with the condition that 0 is reflecting regular and $\left(X_{t}\right)$ is killed as soon as it hits 1 . The transition probabilities $P_{t}(x, A)$ of $X$ have densities $p(t, x, y)$ with respect to $d m$ :

$$
P_{t}(x, A)=\int_{A} p(t, x, y) m(d y) .
$$

Let $\tilde{X}:=\left(\tilde{X}_{t}\right)_{t \geq 0}$ be the corresponding to $X$ space-time process, i.e. a Markov standard process on $\widetilde{E}:=E \times(0, \infty)$ with the transition probabilities $\widetilde{P}_{t}$ generated by

$$
\widetilde{P}_{t}((x, s), A \times \Gamma)=P_{t}(x, A) \cdot \chi_{\Gamma}(s+t) \quad\left((x, s) \in \widetilde{E}, t>0, A \in \mathfrak{B}_{E}, \Gamma \in \mathfrak{B}_{(0, \infty)}\right),
$$

where $\chi_{\Gamma}$ denotes the indicator function of $\Gamma$ and where $\mathfrak{B}_{G}$ is the trace of the $\sigma$-algebra of Borel subsets of $R_{1}$ on $G\left(G \subseteq R_{1}\right)$. Furthermore let $\tilde{\widetilde{X}}$ be the Markov standard process on $\widetilde{E}$ with the transition probabilities $\widetilde{P}_{t}$ generated by

$$
\begin{aligned}
& \widetilde{P}_{t}((x, s), A \times \Gamma)=P_{t}(x, A) \chi_{\Gamma}(s-t) \\
& \left((x, s) \in \widetilde{E}, t>0, A \in \mathfrak{B}_{E}, \Gamma \in \mathfrak{B}_{(, 0 \infty)}\right) .
\end{aligned}
$$

$\tilde{\widetilde{X}}$ is called the coprocess of $\tilde{X}$. The operators $\widetilde{\widetilde{T}}_{t}$ and $\widetilde{T}_{t}$ acting on positive (and on bounded) measurable functions are defined by

$$
\widetilde{T}_{t} f(x):=\int f(y, s+t) P(t, x, d y) \quad(\tilde{x}=(x, s) \in \widetilde{E})
$$

and

$$
\widetilde{T}_{t} f(x):=\int f(y, s-t) P(t, x, d y) \quad(t<s),=0 \quad(t \geqq s) \quad(\tilde{x}=(x, s) \in \tilde{E}) .
$$

In the following the elements of $\widetilde{E}$ always are denoted by $\tilde{x}, \tilde{y}$ etc. with $\tilde{x}=(x, s)$, $\tilde{y}=(y, t)$ etc.

The resolvent kernels

$$
\widetilde{R}_{\lambda}(\tilde{x}, \widetilde{A})=\int_{0}^{\infty} e^{-\lambda u} \widetilde{P}_{u}(\tilde{x}, \widetilde{A}) d u
$$


and

$$
\widetilde{\widetilde{R}}_{\lambda}(\tilde{x}, \tilde{A})=\int_{0}^{\infty} e^{-\lambda u} \widetilde{P}_{u}(\tilde{x}, \tilde{A}) d u \quad(\tilde{x} \in \widetilde{E}, \tilde{A} \text { Borel subset of } \tilde{E}, \lambda>0)
$$

of $\tilde{X}$ and $\tilde{\widetilde{X}}$ respectively are absolutely continuous with respect to $d \tilde{m}:=d m d t$ on $\widetilde{E}$ and have the densities

and

$$
\tilde{r}_{\lambda}(\tilde{x}, \tilde{y})=e^{-\lambda(t-s)} p(t-s, x, y) \chi_{(0, \infty)}(t-s)
$$

$$
\tilde{r}_{\lambda}(\tilde{x}, \tilde{y})=\tilde{r}_{\lambda}(\tilde{y}, \tilde{x}) \quad(\tilde{x}, \tilde{y} \in \tilde{E}, \lambda>0)
$$

respectively. A nonnegative nearly Borel function $f$ on $\widetilde{E}$ is called excessive with respect to $\tilde{X}$ (shortly said excessive) if

$$
\lambda \widetilde{R}_{\lambda} f \leqq f \quad(\lambda>0), \lim _{\lambda \rightarrow \infty} \lambda \widetilde{R}_{\lambda} f=f
$$

pointwise. An excessive function $f$ with $\lim _{\lambda \rightarrow 0} \lambda \widetilde{R}_{\lambda} f=0$ is called purely excessive.

Let $f$ be excessive. If for every $\tilde{x}=(x, s) \in \tilde{E}$ and every open subset $\tilde{A}$ of $\widetilde{E}$ with compact closure in $\widetilde{E}$ we have

$$
f(\tilde{x})=E_{x} f\left(X_{\tau \tilde{A}}, s+\tau \tilde{A}\right) \quad\left(\tau \tilde{A}:=\inf \left\{t>0 \mid \tilde{X}_{t} \notin \tilde{A}\right\}\right)
$$

then $f$ is said to be a harmonic function for $\tilde{X}$ or a parabolic function for $X$ (shortly said a parabolic function). The excessive (harmonic etc.) functions for $\tilde{\tilde{X}}$ will be called coexcessive (coharmonic etc.) for $\tilde{X}$ or shortly coexcessive (coharmonic etc.).

One can show (see e.g. [8], [13]) that a continuous function $h$ on $\widetilde{E}$ with continuous derivatives $\frac{\partial}{\partial t} h$ and $D_{m} D_{p} h$ satisfying $\frac{\partial}{\partial t} h+D_{m} D_{p} h=0 \quad\left(\frac{\partial}{\partial t} h-\right.$ $D_{m} D_{p} h=0$ ) on $\widetilde{E}$ is parabolic (coparabolic). In particular if $\mu \geqq \lambda_{0}$ the function

$$
\tilde{f}_{\mu}(\tilde{x}):=e^{-\mu s} \varphi(x, \mu) \quad(\tilde{x} \in \tilde{E})
$$

is parabolic and the function

$$
\tilde{\tilde{f}}_{\mu}(x):=e^{\mu s} \varphi(x, \mu) \quad(\tilde{x} \in \widetilde{E})
$$

is coparabolic.

The process $\tilde{\tilde{X}}$ starting in $\tilde{x}=(x, s)$ is killed at time $s$. This implies

Proposition 1. Every coexcessive function is purely coexcessive.

Proof. If $f$ is coexcessive, we have for $\lambda>0$ 


$$
\begin{aligned}
& \lambda \widetilde{\widetilde{R}}_{\lambda} f(\tilde{x})=\lambda \int_{0}^{s} \int_{0}^{1} e^{-\lambda(s-t)} p(s-t, y, x) f(y, t) m(d y) d t \\
& =\lambda \int_{0}^{s} e^{-\lambda(s-t)}\left(T_{s-t} f(\cdot, t)\right)(x) d t \leqq f(\tilde{x}) \quad(\tilde{x} \in \widetilde{E})
\end{aligned}
$$

by definition. Thus the integral $\int_{0}^{s}\left(P_{s-t} f(\cdot, t)\right)(x) d t$ exists and hence we have $\lambda \widetilde{\widetilde{R}}_{\lambda} f(\tilde{x}) \rightarrow 0$ for $\lambda \rightarrow 0$.

Especially $\tilde{\tilde{f}}_{\mu}$ is purely coexcessive for every $\mu \geqq \lambda_{0}$. But generally $\tilde{f}_{\mu}$ is not purely excessive e.g. if 1 is entrance then $\tilde{f}_{\lambda_{0}} \equiv 1$ and the identity $\lambda \widetilde{R}_{\lambda} \tilde{f}_{\lambda_{0}}=\tilde{f}_{\lambda_{0}}$ holds.

The application of the theory of Martin boundaries to $X$ requires at first to check that $X$ satisfies some basic assumptions of this theory. These are the so called conditions $K W$ (see [10]) which can be formulated in our case as follows:

(i) $\widetilde{R}_{0}(\cdot, \tilde{K})$ is bounded for every compact subset $\tilde{K}$ of $\tilde{E}$,

(ii) $\lim _{\lambda \rightarrow \infty} \lambda \widetilde{\widetilde{R}}_{\lambda} f(\tilde{x})=f(\tilde{x})(\tilde{x} \in \widetilde{E})$ holds for every $f \in C_{c}(\widetilde{E}):=\{f \mid f$ real-valued continuous function on $\widetilde{E}$ having compact support\},

(iii) $\widetilde{\widetilde{R}}_{\lambda} f$ is continuous and bounded for every bounded real-valued Borelfunction $f$ on $\widetilde{E}$ having compact support and for every $\lambda \geqq 0$,

(iv) $\int_{\widetilde{E}} f \widetilde{R}_{\lambda} g d \tilde{m}=\int_{\widetilde{E}} g \widetilde{\widetilde{R}}_{\lambda} f d \tilde{m}(\lambda \geqq 0 ; f, g$ positive Borel-functions on $\widetilde{E})$.

Proposition 2. The space-time process $\tilde{X}$ satisfies the condition $K W$.

Proof. (i): If $\widetilde{K}$ is compact there exist constants $l, u, v$ with $0<l<1$; $0<u<v<\infty$ such that $\widetilde{K} \subset[0, l] \times[u, v]$. Thus

$$
\widetilde{R}_{0}(\tilde{x}, \tilde{K}) \leqq v-u \quad(\tilde{x} \in \widetilde{E}) .
$$

(ii): Let $f \in C_{c}(\widetilde{E})$. Using the uniform continuity of $f$ it follows that for every $\varepsilon>0$ there exists $a \delta>0$ such that

$$
|f(x, t)-f(x, s)|<\varepsilon \quad(x \in E, t \in(s-\delta, s), s>0) .
$$

Because $\left(T_{h}\right)_{h \geq 0}$ is a strongly continuous semigroup, for every $\varepsilon>0$ there exists a $\delta_{1}>0$ such that

$$
\left|\left(T_{h} f(\cdot, s)\right)(x)-f(x, s)\right|<\varepsilon \quad\left(x \in E, s>0, h<\delta_{1}\right) .
$$

Summarizing these remarks it follows

$$
\left|\left(T_{h} f(\cdot, t)\right)(x)-f(x, s)\right|<2 \varepsilon \quad\left(x \in E, s>\delta, t \in(s-\delta, s), h<\delta_{1}\right),
$$


and thus we have

$$
\begin{aligned}
\lim _{\lambda \rightarrow \infty} \lambda \widetilde{\widetilde{R}}_{\lambda} f(\tilde{x})=\lim _{\lambda \rightarrow \infty} \lambda \int_{0}^{s-\delta_{1}} e^{-\lambda(s-t)}\left(T_{s-t} f(\cdot, t)\right)(x) d t \\
\quad+\lim _{\lambda \rightarrow \infty} \lambda \int_{s-\delta_{1}}^{s} e^{-\lambda(s-t)}\left(T_{s-t} f(\cdot, t)\right)(x) d t \\
\quad=\lim _{\lambda \rightarrow \infty} \lambda \int_{s-\delta_{1}}^{s} e^{-\lambda(s-t)}\left(T_{s-t} f(\cdot, t)\right)(x) d t=f(x) .
\end{aligned}
$$

(iii): It suffices to prove (iii) for $f=\chi_{\tilde{A} \cap \tilde{E}}$, where $\tilde{A}=[0, l] \times[u, v]$ is a compact rectangle of $I \times(0, \infty)$. Let $f$ be of such type and suppose $\left(x_{0}, s_{0}\right),\left(x_{1}, s_{1}\right) \in \widetilde{E}$. Then

$$
\left|\widetilde{\widetilde{R}}_{\lambda} f\left(x_{0}, s_{0}\right)-\widetilde{\widetilde{R}}_{\lambda} f\left(x_{1}, s_{1}\right)\right| \leqq \iint_{\tilde{A}}\left|p\left(s_{0}-t, x_{0}, y\right)-p\left(s_{1}-t, x_{1}, y\right)\right| m(d y) d t .
$$

If $v<s_{0}$ the right side tends to zero for $\left(x_{1}, s_{1}\right) \rightarrow\left(x_{0}, s_{0}\right)$ because $p(h, x, y)$ is uniformly continuous on $\left[h_{0}, h_{1}\right] \times[0, l]^{2}\left(0<h_{0}<h_{1}<\infty, l<1\right)$.

If $v<s_{0}$ the right side of the inequality can be written as $\int_{u}^{s_{0}-\eta} \int_{0}^{l} \cdots$ $\cdots m(d y) d t+\int_{s_{0}-\eta}^{s_{0}} \int_{0}^{l} \cdots m(d y) d t$ for every $\eta$ with $0<\eta<s_{0}$. The first integral tends to zero for $\left(x_{1}, s_{1}\right) \rightarrow\left(x_{0}, s_{0}\right)$ as we have shown above. The second is bounded by $2 \eta$. Thus (iii) is proved.

(iv): Using the definition of $\widetilde{R}_{\lambda}$ and $\widetilde{\widetilde{R}}_{\lambda}$ the proof follows by an easy calculation.

\section{§2. A Martin Boundary for the Space-Time Process}

At first let us define Martin boundaries for the space-time process $X$ following the general line of Meyer [9]. The boundary very depends on the so called Martin kernel used for the construction, i.e. on a renormed resolvent kernel. To construct a Martin boundary for $X$ which describes sufficiently many excessive functions, e.g. the bounded ones, it is necessary to renorm the resolvent kernels by a suitable purely coexessive function. Therefore we define

$$
\begin{aligned}
& n(\bar{y}):=\tilde{\tilde{f}_{\lambda_{0}}}(\tilde{y})=e^{\lambda_{0} t} \varphi\left(y, \lambda_{0}\right), \quad d \zeta(y):=n(\tilde{y}) m(d \tilde{y}), \\
& k_{\lambda}(\tilde{x}, \tilde{y}):=\frac{\tilde{g}_{\lambda}(\tilde{x}, \tilde{y})}{n(\tilde{y})}, \\
& \tilde{K}_{\lambda}(\tilde{x}, d \tilde{y}):=k_{\lambda}(\tilde{x}, \tilde{y}) \zeta(d \tilde{y})=\widetilde{R}_{\lambda}(\tilde{x}, d \tilde{y}), \\
& \tilde{\widetilde{K}}_{\lambda}(\tilde{x}, d \tilde{y}):=k_{\lambda}(\tilde{y}, \tilde{x}) \zeta(d \tilde{y})=\frac{n(\tilde{y})}{n(\tilde{x})} \widetilde{\widetilde{R}}_{\lambda}(\tilde{x}, d \tilde{y}) \quad(\lambda \geqq 0, \tilde{x}, \tilde{y} \in \tilde{E}) .
\end{aligned}
$$


We remark $n(\tilde{y}) \equiv 1$ if the boundary 1 is entrance.

Let $d_{0}$ be a metric on $\widetilde{E}$ generating the usual topology on $\widetilde{E}$.

Definition ([9]). A compact metric space $(\tilde{F}, \tilde{d})$ is called a Martin compact for $\tilde{X}$ if

(a): $\widetilde{E}$ is a dense subset of $\widetilde{F}$ such that the injection from $\left(\widetilde{E}, d_{0}\right)$ into $(\widetilde{F}, \tilde{d})$ is continuous,

(b): for every $f \in C_{c}(\widetilde{E})$ the function $\tilde{\widetilde{K}}_{0} f$ can be extended to a continuous function on $\widetilde{F}$, again denoted by $\widetilde{\widetilde{K}}_{0} f$.

The set $\widetilde{F}^{\prime}:=\widetilde{F} \backslash \widetilde{E}$ is called a Martin boundary of $\widetilde{X}$.

For any $\tilde{y} \in \tilde{F}$ by $f \rightarrow \widetilde{\widetilde{K}}_{0} f(\tilde{y})\left(f \in C_{c}(\tilde{E})\right)$ a Radon measure on $\widetilde{E}$ is given which is absolutely continuous with respect to $d \zeta$. The corresponding density for $\tilde{y} \in \widetilde{E}$ coincides with $k_{0}(\cdot, \tilde{y})=: k_{\tilde{y}}(\cdot)$ and is denoted for $\tilde{y} \in \widetilde{F}^{\prime}$ also by $k_{\tilde{y}}(\cdot)$. For every $\tilde{y} \in \widetilde{F}$ the function $k_{\tilde{y}}\left({ }^{\circ}\right)$ is excessive ([10]). Let $d_{1}$ be a metric on $\widetilde{E}$ such that a sequence $\left(\tilde{x}_{n}\right)$ of points $\tilde{x}_{n} \in \widetilde{E}$ is a $d_{1}$-Cauchy sequence if and only if either $\left|x_{n}-x_{m}\right|+\left|s_{n}-s_{m}\right| \rightarrow 0(n, m \rightarrow \infty)$ or $s_{n} \rightarrow \infty(n \rightarrow \infty)$ independent of $x_{n}$. It is clear that the completion of the metric space $\left(\widetilde{E}, d_{1}\right)$ is equal (up to isomorphism) to $\widetilde{F}:=(F \times[0, \infty)) \cup\{(1 . \infty)\}$ with the extension $\tilde{d}_{1}$ of $d_{1}$ to $\widetilde{F}$.

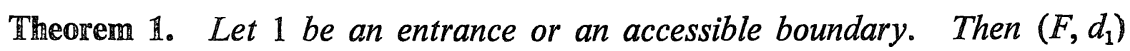
is a Martin compact for the space-time process $X$.

Proof. It is clear that $\left(\tilde{F}, \tilde{d_{1}}\right)$ is compact and that the point (a) of the definition above is satisfied. Thus we shall prove only (b).

Let $f \in C_{c}(\widetilde{E})$. Thus there exist numbers $l, u, v$ with $0<l<1,0<u<v<\infty$ such that $f$ vanishes outside of $[0, l] \times[u, v]$. We will prove that

$$
\widetilde{\widetilde{K}}_{0} f(\tilde{y})=\int_{0}^{1} \int_{0}^{t} p(t-s, x, y) \frac{\varphi\left(x, \lambda_{0}\right)}{\varphi\left(y, \lambda_{0}\right)} e^{-\lambda_{0}(t-s)} f(x, s) m(d x) d s \quad(\tilde{y} \in \widetilde{E})
$$

has a continuous extension to $\widetilde{F}$ and, moreover, we shall determine the densities $k_{\tilde{y}_{0}}(\cdot)$ for $\tilde{y}_{0} \in \widetilde{F}^{\prime}$. To this purpose we consider three cases.

First Case: Let $\tilde{y}_{0}=\left(y_{0}, 0\right)$ for some $y_{0} \in F$. Then $\widetilde{\widetilde{K}}_{0} f(\tilde{y})=\widetilde{\widetilde{K}}_{0} f(y, t)=0$ in neighbourhood of $\tilde{y}_{0}$ (namely if $t<u$ ). Thus $\tilde{\widetilde{K}}_{0} f$ can be continuous extended to $\tilde{y}_{0}$ and it holds

$$
k_{\tilde{y}_{0}}(\tilde{x})=0 \quad\left(\tilde{y}_{0}=\left(y_{0}, 0\right), y_{0} \in F ; \tilde{x} \in \widetilde{E}\right) .
$$

Second Case: Let $\tilde{y}_{0}=\left(1, t_{0}\right)$ for some $t_{0} \in(0, \infty)$. Firstly we suppose 1 is entrance. Then $\lambda_{0}=0$ and $\varphi\left(\bullet, \lambda_{0}\right)=1$. From [5] Theorem 1 follows the 
identity

$$
\lim _{y \rightarrow 1} \int_{0}^{1} p\left(t_{0}-s, x, y\right) f(x, s) m(d x)=\int_{0}^{1} p\left(t_{0}-s, x, 1\right) f(x, s) m(d x)
$$

for every $s<t_{0}$, where $p(h, x, 1)$ is an uniquely determined strictly positive continuous function of $(x, h) \in \widetilde{E}$.

The integrals on the left side of (3) are dominated by $C:=\max |f(\tilde{z})|<\infty$. Integrating (3) with respect to $s$ and using Fubini's theorem and Lebesgue's dominated convergence theorem it follows for $t>0$

$$
\lim _{y \rightarrow 1} \widetilde{\widetilde{K}}_{0} f(y, t)=\int_{0}^{1} \int_{0}^{t} p(t-s, x, 1) f(x, s) m(d x) d s .
$$

Now we shall estimate $\widetilde{\widetilde{K}}_{0} f(y, t)-\widetilde{\widetilde{K}}_{0} f\left(y, t_{0}\right)$. We can assume $t<t_{0}$. Then this difference is equal to

$$
\begin{gathered}
\int_{0}^{1} \int_{0}^{t}\left(p(t-s, x, y)-p\left(t_{0}-s, x, y\right)\right) f(x, s) d s m(d x) \\
-\int_{0}^{1} \int_{t}^{t_{0}} p\left(t_{0}-s, x, y\right) f(x, s) d s m(d x) .
\end{gathered}
$$

The absolute value of the first integral can be estimated by $\int_{0}^{t} \mid\left(T_{t_{0}-t} f(\circ, s)\right)$ $(y)-f(y, s) \mid d s$. The strong continuity of $\left(T_{h}\right)$ implies $\left(T_{t_{0}-i} f(\circ, s)\right)(y)-f(y, s)$ $\underset{t \rightarrow t_{0}}{\longrightarrow} 0$ uniformly in $y$ for every $s$. Thus by Lebesgue's dominated convergence theorem the first integral tends to zero for $t \rightarrow t_{0}$.

The second integral vanishes for $t-t_{0}$ uniformly in $y$ because $1 \int_{0}^{1} p\left(t_{0}-s\right.$, $x, y) f(x, s) m(d x) \mid \leqq C<\infty$ uniformly in $x \in E$ and $s \leqq t_{0}$.

Summarizing we obtain under the assumption that 1 is entrance

$$
\lim _{\tilde{y} \rightarrow \tilde{y}_{0}} \tilde{\widetilde{K}}_{0} f(\tilde{y})=\int_{0}^{1} \int_{0}^{t_{0}} p\left(t_{0}-s, x, 1\right) f(x, s) m(d x) d s
$$

and

$$
\begin{aligned}
& \left.k_{\tilde{y}_{0}}(x)=p\left(t_{0}-s, x, 1\right) \chi_{(0, \infty)}\left(t_{0}-s\right)\right) \\
& \left(\tilde{x}=(x, s) \in \widetilde{E}, y_{0}=\left(1, t_{0}\right), t_{0} \in(0, \infty)\right) .
\end{aligned}
$$

Now let 1 be accessible. Then by $d m^{*}=\varphi^{2}\left(\cdot, \lambda_{0}\right) d m$ and $d p^{*}=\varphi^{-2}\left(\circ, \lambda_{0}\right) d p$ a new speed measure $m^{*}$ and a new scale $p^{*}$ on $[0,1)$ are given. The boundary 1 is entrance for $\left(m^{*}, p^{*}\right)$ (see [5]) and the corresponding transition density is

$$
p^{*}(h, x, y)=\frac{e^{-\lambda_{0} h} p(h, x, y)}{\varphi\left(x, \lambda_{0}\right) \varphi\left(y, \lambda_{0}\right)} \quad(x, y \in E, h>0) .
$$


Applying the preceding results to $\left(m^{*}, p^{*}\right)$ it follows

$$
\begin{aligned}
\lim _{y \rightarrow 1} & \int_{0}^{1} p(t-s, x, y) e^{-\lambda_{0}(t-s)} \frac{\varphi\left(x, \lambda_{0}\right)}{\varphi\left(y, \lambda_{0}\right)} f(x, s) m(d x) \\
& =\int_{0}^{1} p^{*}(t-s, x, 1) \varphi^{2}\left(x, \lambda_{0}\right) f(x, s) m(d x) \quad(0<s<t) .
\end{aligned}
$$

We know from [5] that $D_{p} \varphi\left(1, \lambda_{0}\right)=\lim _{x \rightarrow 1} \frac{\varphi\left(x, \lambda_{0}\right)}{p(x)-p(1)}$ exists and is finite and negative. Defining

$$
D_{p} p(h, x, 1):=p^{*}(h, x, 1) e^{\lambda_{0} h} \varphi\left(x, \lambda_{0}\right) D_{p} \varphi\left(1, \lambda_{0}\right) \quad(h>0, x \in E),
$$

the right side of (5) can be written as

$$
\int_{0}^{1} e^{-\lambda_{0}(t-s)} \varphi\left(x, \lambda_{0}\right) D_{p} p(t-s, x, 1) f(x, s) m(d x) \cdot\left(D_{p} \varphi\left(1, \lambda_{0}\right)\right)^{-1},
$$

and on an analogous way as above it can be shown

$$
\lim _{\tilde{y} \rightarrow \tilde{y}_{0}} \tilde{\widetilde{K}}_{0} f(y)=\left(D_{p} \varphi\left(1, \lambda_{0}\right)\right)^{-1} \int_{0}^{1} \int_{0}^{t_{0}} e^{-\lambda_{0} t_{0}} D_{p} p\left(t_{0}-s, x, 1\right) d \zeta(\tilde{x})
$$

and

$$
\begin{gathered}
k_{\tilde{y}_{0}}(\tilde{x})=e^{-\lambda_{0} t_{0}} \frac{D_{p} p\left(t_{0}-s, x, 1\right)}{D_{p} \varphi\left(1, \lambda_{0}\right)} \chi_{(0 \infty)}\left(t_{0}-s\right) \\
\left(\tilde{x}=(x, s) \in \widetilde{E}, \tilde{y}_{0}=\left(1, t_{0}\right), t_{0} \in(0, \infty)\right)
\end{gathered}
$$

under the assumption that 1 is accessible.

Third Case: $\tilde{y}_{0}=(1, \infty)$. If 1 is entrance, from [5], Theorem 3 it follows the identity

$$
\lim _{t \rightarrow \infty} \int_{0}^{1} \int_{0}^{t} p(t-s, x, y) f(x, s) d s m(d x)=(m(1))^{-1} \int_{0}^{1} \int_{0}^{\infty} f(x, s) d s m(d x) .
$$

This implies

$$
k_{\tilde{y}_{0}}(\tilde{x})=(m(1))^{-1} \quad(\tilde{x} \in \widetilde{E}) .
$$

If 1 is accessible by the same transformation and the same method as in the second case it follows

$$
\lim _{t \rightarrow \infty} \tilde{\widetilde{K}}_{0} f(\tilde{y})=\left(\int_{0}^{1} \varphi^{2}\left(x, \lambda_{0}\right) m(d x)\right)^{-1} \int_{0}^{1} \int_{0}^{\infty} \varphi\left(x, \lambda_{0}\right) f(x, s) e^{-\lambda_{0} s} d \zeta(\tilde{x})
$$

and

$$
k_{\tilde{y}_{0}}(x)=\left(\int_{0}^{1} \varphi^{2}\left(x, \lambda_{0}\right) m(d x)\right)^{-1} e^{-\lambda_{0} s} \varphi\left(x, \lambda_{0}\right) \quad(\tilde{x} \in \widetilde{E}) .
$$


Thus Theorem 1 is proved.

Corollary. Let $\int_{0}^{1} p^{2} d m<\infty$, i.e. $(m, p)$ is in the limit circle case (see e.g. [4], [5]). Then we have an uniformly converging spectral expansion for $p(h, x, 1)$ (if 1 is intrance) and for $D_{p} p(h, x, 1)$ (if 1 is accessible) (see [5], Theorem 2).

Hence we can give such an expansion for $k_{y_{0}}$ :

$$
\begin{gathered}
k_{\tilde{y}_{0}}(\tilde{x})=\left(\frac{1}{m(1)}+\sum_{k=1}^{\infty} e^{\lambda_{k}\left(t_{0}-s\right)} \varphi\left(x, \lambda_{k}\right) \varphi\left(1, \lambda_{k}\right) \tau_{k}\right) \chi_{(0, \infty)}\left(t_{0}-s\right) \\
\left(\tilde{x} \in \tilde{E}, \tilde{y}_{0}=\left(1, t_{0}\right), t_{0} \in(0, \infty)\right)
\end{gathered}
$$

if 1 is entrance with $\int_{0}^{1} p^{2} d m<\infty$ and

$$
\begin{aligned}
k_{\tilde{y}_{0}}(\tilde{x})= & \left(e^{-\lambda_{0} s} \varphi\left(x, \lambda_{0}\right) \tau_{0}\right. \\
& \left.+\sum_{k=1}^{\infty} e^{\lambda_{k}\left(t_{0}-s\right)} \varphi\left(x, \lambda_{k}\right) \frac{D_{p} \varphi\left(1, \lambda_{k}\right)}{D_{p} \varphi\left(1, \lambda_{0}\right)} \cdot e^{-\lambda_{0} t_{0}} \tau_{k}\right) \chi_{(0, \infty)}\left(t_{0}-s\right)
\end{aligned}
$$

$\left(\tilde{x} \in \widetilde{E}, \tilde{y}_{0}=\left(1, t_{0}\right), t_{0} \in(0, \infty)\right)$ if 1 is accessible with $\int_{0}^{1} p^{2} d m<\infty$ (i.e. regular), where the series in (9) and (10) are uniformly converging in $t \geqq t_{0}>0$ and $x \in[0,1)$.

Theorem 2 below gives an integral representation of $\tilde{X}$-excessive and -parabolic functions. To formulate it we need some more notations and propositions from the theory of Martin boundaries which will now be given (see [10], also [7]).

Let $f$ be excessive. From the mentioned theory it is known that the limit

$$
L(f):=\lim _{\lambda \rightarrow \infty} \lambda \int_{\tilde{E}}\left(1-\widetilde{\widetilde{K}}_{\lambda} 1\right)(\tilde{y}) f(\tilde{y}) \zeta(d \tilde{y})
$$

exists (possibly infinite). A short calculation using the definition of $\widetilde{\widetilde{K}}_{\lambda}$ and $\zeta$ in our case implies

$$
L(f)=\lim _{\lambda \rightarrow \infty} \lambda \int_{0}^{\infty} e^{-\lambda s}\left(\int_{0}^{1} f(x, s) \varphi\left(x, \lambda_{0}\right) m(d x)\right) d s .
$$

Because $f$ is excessive we have

Thus

$$
\left(T_{h} f(\cdot, t+h)\right)(x) \leqq f(x, t) \quad(h, t>0, x \in E) .
$$

$$
\begin{aligned}
& \int_{0}^{1} e^{h \lambda_{0}} f(y, t+h) \varphi\left(y, \lambda_{0}\right) m(d y)=\int_{0}^{1} f(y, t+h)\left(T_{h} \varphi\left(\cdot, \lambda_{0}\right)\right)(y) m(d y) \\
= & \int_{0}^{1}\left(T_{h} f(\cdot, t+h)\right)(x) \varphi\left(x, \lambda_{0}\right) m(d x) \leqq \int_{0}^{1} f(x, t) \varphi\left(x, \lambda_{0}\right) m(d x) \quad(h, t>0) .
\end{aligned}
$$


Therefore the function $h \rightarrow \int_{0}^{1} e^{\lambda_{0} h} f(y, t+h) \varphi\left(y, \lambda_{0}\right) m(d y)$ decreases if $t$ is fixed and $h$ increases. Hence the function $h \rightarrow e^{-\lambda_{0} h} \int_{0}^{1} f(y, t-h) \varphi\left(y, \lambda_{0}\right) m(d y)$ increases if $h$ increases from 0 to $t$ for every $t>0$. Thus the limit $\lim _{h \uparrow 0} \int_{0}^{1} f(y, h) \varphi\left(y, \lambda_{0}\right) m(d y)$ exists and by properties of the Laplace-transformation we have

$$
L(f)=\lim _{h \downarrow 0} \int_{0}^{1} f(y, h) \varphi\left(y, \lambda_{0}\right) m(d y)
$$

with $L(f) \in(0, \infty]$ if $f$ is excessive and non identical zero. In the theory of Martin boundaries every excessive function $f$ satisfying a certain condition (e.g. $L(f)<\infty$ in our case) is represented by minimal excessive functions (see below). An essential point is to choose the purely coexcessive function $n$ in the definition of $\widetilde{K}_{\lambda}$ in such a way that as many as possible excessive functions $f$ satisfy the mentioned condition. Here we have taken $n(y, t)=\varphi\left(y, \lambda_{0}\right) e^{+\lambda_{0} t}$. This implies e.g. that the bounded excessive functions and the functions $\tilde{f}_{\mu}(x, s)=e^{-\mu s} \varphi(x, \mu)\left(\mu \geqq \lambda_{0}\right)$ are included if 1 is entrance or accessible because $\varphi\left(\cdot, \lambda_{0}\right)$ is bounded and $m$-integrable and $\varphi\left(\cdot, \lambda_{0}\right)$ is bounded (if 1 is accessible) or $m$-integrable (if 1 is entrance) (see [5]).

An excessive function $f=0$ is called extreme if for every two excessive functions $f_{1}, f_{2}$ with $f=\alpha f_{1}+(1-\alpha) f_{2}$ for some $\alpha \in(0,1)$ it follows $f=f_{1}=f_{2}$.

We say that the point $\tilde{y} \in \widetilde{F}$ is a minimal point if $\tilde{y} \in \widetilde{E}$ or $\tilde{y} \in \widetilde{F}_{m}^{\prime}:=$ $\left\{\tilde{z} \in \widetilde{F}^{\prime} \mid L\left(k_{\tilde{z}}\right)=1, k_{\tilde{z}}\right.$ is extreme and parabolic\}. The set of minimal points of $\widetilde{F}$ is a Borel set and we denote it by $\widetilde{F}_{m}$. (Using the fact that for the process $\widetilde{X}$ considered here the function $k_{\tilde{y}}$ for no $\tilde{y} \in \widetilde{E}$ is harmonic (the proof is not difficult and omitted here) one can show that the definition given above coincides with the definition of minimal points given in [10]. See also [7].)

The functions $k_{\tilde{y}}\left(\tilde{y} \in \widetilde{F}_{m}\right)$ are also called minimal.

As already said, from the theory of Martin boundaries it follows that for any excessive function $f$ with $L(f)=1$ there exists a Radon measure $\mu_{f}$ on $\widetilde{F}_{1}:=\left\{\tilde{y} \in \widetilde{F} \mid L\left(k_{\tilde{y}}\right)=1\right\}$ with $\mu_{f}\left(\widetilde{F}_{1}\right)=1$ such that

$$
f(\tilde{x})=\int_{\widetilde{F}} k_{\tilde{y}}(\tilde{x}) \mu_{f}(d \tilde{y}) \quad(\tilde{x} \in \tilde{E})
$$

(see [10]).

If the functions $\widetilde{\widetilde{K}}_{0} g\left(g \in C_{c}(\widetilde{E})\right)$ separate the points of $\widetilde{F}_{m}$, then there exists an uniquely determined measure $\mu_{f}$ supported on $\widetilde{F}_{m}$ such that (12) holds.

Now we are ready to formulate and prove a representation theorem for 
quasidiffusion-space-time excessive functions $f$ satisfying $L(f)<\infty$.

Theorem 2. Let 1 be an entrance or an accessible boundary.

Then we have:

(i): The set $\widetilde{F}_{m}$ of minimal points of $\widetilde{F}$ is equal to

$$
\widetilde{E} \cup\left\{\left(1, t_{0}\right) \mid t_{0} \in(0, \infty]\right\},
$$

(ii): For every excessive function $f$ with $L(f)=1$ there exists an uniquely determined measure $\mu_{f}$ on $\widetilde{F}_{m}$ such that

$$
\mu_{f}\left(\widetilde{F}_{m}\right)=1 \quad \text { and } \quad f(\tilde{x})=\int_{\tilde{E}} k_{\tilde{y}}(\tilde{x}) \mu_{f}(d \tilde{y}) \quad(\tilde{x} \in \widetilde{E})
$$

(iii): For every parabolic function $f$ with $L(f)=1$ there exists an uniquely determined measure $\mu_{f}$ on $(0, \infty]$ such that

$$
\mu_{f}((0, \infty])=1, f(\tilde{x})=\int_{0+}^{\infty+} k_{(1, t)}(\tilde{x}) \mu_{f}(d t) \quad(\tilde{x} \in \widetilde{E})
$$

and

$$
\mu_{f}((0, s])=-D_{p} \varphi\left(1, \lambda_{0}\right) \lim _{x \rightarrow 1} \int_{0}^{s} e^{\lambda_{0} t} f(x, t) d t \quad(s \in(0, \infty))
$$

if 1 is accessible,

$$
\mu_{f}((0, s])=\lim _{x \rightarrow 1}(p(x))^{-1} \int_{0}^{x} f(x, t) d t \quad(s \in(0, \infty))
$$

if 1 is entrance.

Proof. At first we remark that the points $\left(y_{0}, 0\right)$ not belong to $\widetilde{F}_{m}$ because $k_{\left(y_{0}, 0\right)} \equiv 0$ (see also (2)). Thus we have the part $\subseteq$ of (i). To prove the part we have to show that every point $\widetilde{y}_{0}=\left(1, t_{0}\right)\left(0<t_{0} \leqq \infty\right)$ belongs to $\widetilde{F}_{m}^{\prime}$. At first suppose $t_{0} \in(0, \infty)$ and let $\tilde{y}_{0}=\left(1, t_{0}\right)$ be fixed. Then $L\left(k_{\tilde{y}_{0}}\right)=$ $\lim _{s \downarrow 0} \int_{0}^{1} p\left(t_{0}-s, y, 1\right) m(d y)=1$ if 1 is entrance and $L\left(k_{\tilde{y}_{0}}\right)=\lim _{s \downarrow 0} \frac{e^{-\lambda_{0} t_{0}}}{D_{p} \varphi\left(1, \lambda_{0}\right)} \int_{0}^{1} D_{p} p\left(t_{0}\right.$ $-s, x, 1) \varphi\left(x, \lambda_{0}\right) m(d x)=\lim _{s \downarrow 0} e^{-\lambda_{0} s} p^{*}\left(t_{0}-s, x, 1\right) m^{*}(d x)=1$ if 1 is accessible. (For notations see the proof of Theorem 1.)

Now we shall show that $k_{\tilde{y}_{0}}$ is parabolic. We have already mentioned that $k_{\tilde{y}_{0}}$ is excessive. Thus only (1) is to prove. If $\tilde{x}=(x, s) \in \widetilde{E}$ with $s \geqq t_{0}$ we have by definition $k_{\tilde{y}_{0}}(\tilde{x})=0$ and therefore $k_{\tilde{y}_{0}}\left(X_{t}, s+t\right)=0$ a.e. with respect to $P_{x}$ for every $t>0$. Thus (1) holds for such $\tilde{x}$.

Let $\tilde{x}=(x, s)$ with $s<t_{0}$ be fixed. To show (1) we can restrict ourselves to 
rectangles of the form

$$
\begin{gathered}
\tilde{A_{\delta, h}}:=\{(z, u)|| x-z \mid<\delta, x \pm \delta \in E, 0<u-s<h\} \\
(\max (\delta, 1-\delta) \quad 1 ; \delta, h>0) .
\end{gathered}
$$

Let 1 be entrance. Then $k_{\tilde{y}_{0}}(\tilde{x})=p\left(t_{0}-s, x, 1\right)=\int_{0}^{1} P_{h}(1, d z) p\left(t_{0}-s-h, x, z\right)$ $\left(s+h<t_{0}\right)$, by definition (see [5]), where $P_{h}(1, d z)$ is the transition function of $X$ at the point 1 . Suppose at first that $\tilde{A}:=\tilde{A_{\delta, h}}$ is a rectangle of the mentioned form with $h<t_{0}-s$. Then we have

$$
\begin{gathered}
E_{x} k_{\tilde{y}_{0}}\left(X_{\tau \tilde{\mathcal{A}}}, s+\tau \tilde{A}\right)=\int_{\partial \tilde{A}} p\left(t_{0}-u, z, 1\right) P_{x}\left(\left(X_{\tau_{\tilde{\mathbb{A}}}}, \tau_{\tilde{A}}+s\right) \in d \tilde{z}\right) \\
=\int_{0}^{1} P_{t_{0}-s-h}(1, d y) \int_{\partial \tilde{A}} p(s+h-u, z, y) P_{x}\left(\left(X_{\tau_{\tilde{A}}}, \tau_{\tilde{A}}+s\right) \in d \tilde{z}\right) \\
(\tilde{z}=(z, u)) .
\end{gathered}
$$

Remarking that for every $(y, s+h)$ the function $k_{(y, s+h)}(z, u):=p(s+h-u$, $z, y)$ is parabolic in $(z, u)$ with $u<s+h$ by continuity and the Kolmogorov equations, it follows that the inner integral is equal to $p(s+h-s, x, y)=p(h, x, y)$. Thus

$$
\begin{aligned}
E_{x} k_{\widetilde{y}_{0}}\left(X_{\tau \widetilde{A}}, s+\tau_{\tilde{A}}\right) & =\int_{0}^{1} P_{t_{0}-s-h}(1, d y) p(h, x, y)=p\left(t_{0}-s, x, 1\right) \\
& =k_{\tilde{y}_{0}}(x, s) \quad((x, s) \in \widetilde{E}) .
\end{aligned}
$$

Now let $\tilde{A}:=A_{\delta, h}$ be a rectangle as above with $h \geqq t_{0}-s$. Fixing $h^{\prime}$ with $0<h^{\prime}<t_{0}-s$ and using the preceding step it follows with the notation $\tilde{A^{\prime}}=\tilde{A_{\delta}, h^{\prime}}$

$$
\left.p\left(t_{0}-s, x, 1\right)=\int_{\partial \tilde{A}^{\prime}} p\left(t_{0}-u, z, 1\right) P_{x}\left(X_{\tau_{\tilde{A}^{\prime}}}, \tau \tilde{A}+s\right) \in d z\right) .
$$

The right hand side splits into three parts, namely the integral about the lines $(x+\delta, s) \cdots\left(x+\delta, s+h^{\prime}\right),(x-\delta, s) \cdots\left(x-\delta, s+h^{\prime}\right)$ and $\left(x-\delta, s+h^{\prime}\right) \cdots\left(x+\delta, s+h^{\prime}\right)$. The integral along the third line tends to zero if $s+h^{\prime} \rightarrow t_{0}$ :

$$
\lim _{h^{\prime} \rightarrow t_{0}-s} \int_{x_{0}-\delta}^{x_{0}+\delta} p\left(t_{0}-s-h^{\prime}, z, 1\right) P_{x}\left(\tau_{\tilde{A}}>h^{\prime}, X_{h^{\prime}} \in d z\right)=0 .
$$

This is proved as follows. The integrals in (17) are less than $\int_{x-\delta}^{x+\delta} p\left(t_{0}\right.$ $\left.s-h^{\prime}, z, 1\right) p\left(h^{\prime}, x, z\right) m(d z)=\int_{x-\delta}^{x+\delta} p\left(h^{\prime}, x, z\right) P_{t_{0}-s-h^{\prime}}(1, d z)$ and this term converges to zero for $h^{\prime} \rightarrow t_{0}-s$ by continuity of the semigroup $\left(T_{h}\right)_{h \geqq 0}$. Therefore from (16) and (17) it follows 


$$
\begin{aligned}
& p\left(t_{0}-s, x, 1\right)=\int_{s}^{t_{0}} p\left(t_{0}-u, x+\delta, 1\right) P_{x}\left(\tau_{\tilde{A}}+s \in d u, X_{\tau_{\tilde{A}}} \geqq x+\delta\right) \\
& \quad+\int_{s}^{t_{0}} p\left(t_{0}-u, x-\delta, 1\right) P_{x}\left(\tau_{\tilde{A}}+s \in d u, X_{\tau_{\tilde{A}}} \leqq x-\delta\right) \\
& \quad=\int_{\partial \tilde{A}} p\left(t_{0}-u, z, 1\right) P_{x}\left(\left(X_{\tau_{\tilde{A}}}, \tau_{\tilde{A}}+s\right) \in d \tilde{z}\right) .
\end{aligned}
$$

Thus we have proved that $k_{\tilde{y}_{0}}(\cdot)$ is parabolic if 1 is entrance. If 1 is accessible, the proof follows by transformation of $(m, p)$ as in the proof of Theorem 1 and applying the preceding result for the entrance boundary.

The proof that $k_{\widetilde{y}_{0}}$ is extreme depends on the following lemmata.

Lemma 1. We have

$$
\lim _{x \rightarrow 1} \int_{0}^{s} k_{\left(1, t_{0}\right)}(x, u) d u=e^{-\lambda_{0} t_{0}}\left(-D_{p} \varphi\left(1, \lambda_{0}\right)\right)^{-1} \chi_{[0, s]}\left(t_{0}\right)
$$

if 1 is accessible and

$$
\lim _{x \rightarrow 1}(p(x))^{-1} \int_{0}^{s} k_{\left(1, t_{0}\right)}(x, u) d u=\chi_{[0, s]}\left(t_{0}\right) \quad(s \in(0, \infty))
$$

if 1 is entrance.

Proof of Lemma 1. Let 1 be accessible. Then by Theorem 2 and Lemma 4 of [5] it follows

$$
\begin{array}{r}
-D_{p} \varphi\left(1, \lambda_{0}\right) e^{\lambda_{0} t_{0}} \int_{0}^{s} k_{\left(1, t_{0}\right)}(x, u) d u=\lim _{y \rightarrow 1} \int_{0}^{t_{0} \wedge s} \frac{p\left(t_{0}-u, x, y\right)}{p(1)-p(y)} \\
= \begin{cases}1-\int_{0}^{1} p\left(t_{0}, x, z\right) m(d z) & \text { if } s \geqq t_{0}, \\
\int_{0}^{1} p\left(t_{0}-s, x, z\right) m(d z)-\int_{0}^{1} p\left(t_{0}, x, z\right) m(d z) & \text { if } s<t_{0} .\end{cases}
\end{array}
$$

Also from the accessibility of 1 it follows $T_{h} 1(x) \rightarrow 0$ for every $h>0$ if $x \rightarrow 1$. Thus we have (18).

Let 1 be entrance. We choose $\lambda>0$ and consider the new speed measure $d m^{(\lambda)}=\varphi^{2}(\cdot, \lambda) d m$ and the new scale $d p^{(\lambda)}=\varphi^{-2}(\cdot, \lambda) d p$. One can show that 1 is accessible with respect to $\left(m^{(\lambda)}, p^{(\lambda)}\right)$ and that the corresponding to $\left(m^{(\lambda)}, p^{(\lambda)}\right)$ transition density is

$$
p^{(\lambda)}(h, x, y)=\frac{p(h, x, y) e^{-h}}{\varphi(x, \lambda) \varphi(y, \lambda)} \quad(x, y \in E, h>0)
$$

(see [5]). Moreover, we have 


$$
k_{\left(1, t_{0}\right)}^{(\lambda)}(x, s)=e^{\lambda t_{0}} \frac{D_{p}^{(\lambda)} p^{(\lambda)}\left(t_{0}-s_{1} x_{1} 1\right)}{D_{p}^{(\lambda)} \varphi^{(\lambda)}(1,-\lambda)} \chi_{(0, \infty)}\left(t_{0}-s\right) \quad((x, s) \in \widetilde{E})
$$

Using (18) it follows

$$
\lim _{x \rightarrow 1}(-1) \int_{0}^{s} D_{p}(\lambda) p^{(\lambda)}\left(t_{0}-u, x, 1\right) d u=\chi_{[0, u]}\left(t_{0}\right)
$$

From Theorem 2 of [5] we have

$$
\begin{gathered}
-\int_{0}^{s} D_{p}(\lambda) p^{(\lambda)}\left(t_{0}-u, x, 1\right) d u=\lim _{y \rightarrow 1} \int_{0}^{s} \frac{p^{(\lambda)}\left(t_{0}-u, x, y\right)}{p^{(\lambda)}(1)-p^{(\lambda)}(y)} d u \\
=\lim _{y \rightarrow 1} \int_{0}^{s} \frac{e^{-\lambda\left(t_{0}-u\right)} p\left(t_{0}-u, x, y\right)}{\varphi(x, \lambda)(\Gamma(\lambda) \varphi(y, \lambda)-\psi(y, \lambda))} d u .
\end{gathered}
$$

where $\psi(\cdot, \lambda)$ is the solution of $D_{m} D_{p} g=\lambda g$ satisfying $\psi(0, \lambda)=0, D_{p}^{-} \psi(0, \lambda)=1$, and $\Gamma(\lambda)=p^{(\lambda)}(1)=\lim _{x \hat{\jmath}_{1}} \frac{\psi(x, \lambda)}{\varphi(x, \lambda)}$. Using known properties of the function $\chi(\cdot, \lambda):=\Gamma(\lambda) \varphi(\cdot, \lambda)-\psi(\cdot, \lambda)$ (see also [5]) it follows that the last integral is equal to

$$
e^{-\lambda t_{0}}(\varphi(x, \lambda) \chi(1, \lambda))^{-1} \int_{0}^{s} e^{\lambda u} p\left(t_{0}-u, x, 1\right) d u .
$$

Thus by using $D_{p} \varphi(1, \lambda) \cdot \chi(1, \lambda)=1$ (this follows from [5] remarking $D_{p} \varphi(1, \lambda)<\infty$, $\chi(1, \lambda)>0)$ we have

$$
\begin{gathered}
e^{-\lambda t_{0}} \lim (p(x))^{-1} \int_{0}^{s} e^{\lambda u} p\left(t_{0}-u, x, 1\right) d u=-1 \\
=-\lim _{x \rightarrow 1} \int_{0}^{s} D_{p}(\lambda) p^{(\lambda)}\left(t_{0}-u, x, 1\right) d u=\chi_{[0, s]}\left(t_{0}\right) \quad \text { for every } \lambda>0 .
\end{gathered}
$$

Hence (19) follows by letting $\lambda \rightarrow 0$. Thus Lemma 1 is proved.

Lemma 2. Let $g$ be a parabolic function with $L(g)=1$ and $g(\tilde{x})=\int_{0+}^{\infty+} k_{(1, t)}(\tilde{x})$ $\times \mu(d t)$ for some finite measure $\mu$ on $(0, \infty]$. Then

$$
-D_{p} \varphi\left(1, \lambda_{0}\right) \lim _{x \rightarrow 1} \int_{0}^{s} e^{\lambda_{0} u} g(x, u) d u=\mu((0, s]) \quad(s<\infty)
$$

if 1 is accessible and

$$
\lim _{x \rightarrow 1}(p(x))^{-1} \int_{0}^{s} g(x, u) d u=\mu((0, s]) \quad(s<\infty)
$$

if 1 is entrance. 
Proof of Lemma 2. Let 1 be accessible. Then by Fubini's theorem $\int_{0}^{s} g(x, u) d u=\int_{0}^{\infty} \int_{0}^{s} k_{(1, t)}(x, u) d u \mu(d t)$. If $x$ converges to 1 , the limitation and integration can be changed. This is seen as follows. From (20) we have

$$
\int_{0}^{s} k_{(1, t)}(x, u) d u \leqq e^{-\lambda_{0} t}\left(-D_{p} \varphi\left(1, \lambda_{0}\right)\right)^{-1} \int_{0}^{1} p(t-s, x, z) m(d z) \quad(t>s, x \in E) .
$$

We show that the continuous function $h \rightarrow e^{-\lambda_{0}{ }^{h}} p(h, x, z) m(d z)$ is bounded for large $h$. This justifies the change in virtue of the Lebesgue's dominated convergence theorem. To this purpose we calculate the Laplace-transform (see [5]):

$$
\lambda \int_{0}^{\infty} e^{-\lambda h} e^{-\lambda_{0} h} d h \int_{0}^{1} p(h, x, z) m(d z)=\frac{\lambda}{\lambda+\lambda_{0}}\left(1-\frac{\varphi\left(x, \lambda+\lambda_{0}\right)}{\varphi\left(1, \lambda+\lambda_{0}\right)}\right) \quad(\lambda>0)
$$

and let $\lambda$ tend to zero. Then the right hand side converges to $-\frac{\varphi\left(x, \lambda_{0}\right)}{\lambda_{0}}$ $\times\left(\left.\frac{\partial \varphi\left(1, \lambda_{0}+\lambda\right)}{\partial \lambda}\right|_{\lambda=0}\right)^{-1}$. For every $x<1$ the function $\lambda \rightarrow \varphi(x, \lambda)$ is entire and has the representation

$$
\varphi(x, \lambda)=\prod_{k=0}^{\infty}\left(1-\frac{\lambda}{\lambda_{k}(x)}\right)
$$

where $\left(\lambda_{k}(x)\right)$ are the zeros of $\varphi\left(x,{ }^{\circ}\right)$ (see [4]).

If $x$ tends to 1 , the numbers $\lambda_{k}(x)$ converge to the points $\lambda_{k}$ of increasing of the main spectral function $\tau$. Because $\sum_{k=1}^{\infty} \frac{1}{\left|\lambda_{k}\right|}<\infty$ the right hand side of (23) tends to the entire function $\prod_{k=1}^{\infty}\left(1-\frac{\lambda}{\lambda_{k}}\right)$ and this limit is equal to $\varphi(1, \lambda)$. Therefore $\left.\frac{\partial \varphi\left(1, \lambda_{0}+\lambda\right)}{\partial \lambda}\right|_{\lambda=0}=-\prod_{k=1}^{\infty}\left(1-\frac{\lambda_{0}}{\lambda_{k}}\right)$ is finite and nonzero. Hence by $\varphi\left(x, \lambda_{0}\right)>0(x \in E)$ it follows that $e^{-\lambda_{0} h} \int_{0}^{1} p(h, x, z) m(d z)$ converges to a finite limit if $h \rightarrow \infty \quad$ Summarizing and using Lemma 1 we obtain

$$
\lim _{x \rightarrow 1} \int_{0}^{s} g(x, u) d u=-\int_{0}^{\infty}\left(D_{p} \varphi\left(1, \lambda_{0}\right)\right)^{-1} \bullet e^{-\lambda_{0} t} \chi_{(0, s)}(t) \mu(d t)
$$

and hence (21) follows.

If 1 is entrance we can transform $(m, p)$ as in the proof of Lemma 1 into a new pair $\left(m^{(\lambda)}, p^{(\lambda)}\right)$ such that 1 is $\left(m^{(\lambda)}, p^{(\lambda)}\right)$-accessible. Applying (21) and transforming back we obtain (22). 
Now we shall prove that $k_{\tilde{y}_{0}}$ is extreme. To this aim we remark that every parabolic function $g$ with $L(g)=1$, has a representation $g=\int_{\tilde{F}_{1}} k_{\tilde{y}} \mu_{g}(d y)$ where the Radon-measure $\mu_{g}$ is supported on $F_{1}^{\prime}$ (see above). (That $\mu_{g}$ is supported on $F_{1}^{\prime}$ follows from the fact that no $k_{\tilde{y}}$ for $\tilde{y} \in \tilde{E}$ is parabolic (see [10]). The last point has to be shown, but this step is easy to see and omitted here.)

Suppose $g_{1}, g_{2}$ are two excessive functions with $\alpha g_{1}+(1-\alpha) g_{2}=k_{\tilde{y}_{0}}$ for some $\alpha \in(0,1)$. Then $g_{1}$ and $g_{2}$ have to be parabolic because $k_{y_{0}}$ it is, and by the preceding remark they have the representation

$$
g_{i}=\int_{0}^{\infty} k_{(1, t)} \mu_{i}(d t)
$$

for some (finite) measure $\mu_{i}$ on $(0, \infty](i=1,2)$.

Using Lemmata 1 and 2 it follows that $k_{\tilde{y}_{0}}$ is extreme. Thus we have proved that every $\tilde{y}_{0}=\left(1, t_{0}\right)$ with $0<t_{0}<\infty$ is a minimal point.

At least we consider $k_{(1, \infty)}(x)=e^{-\lambda_{0} s} \varphi\left(x, \lambda_{0}\right)(\tilde{x} \in \tilde{E})$. This function is parabolic and satisfies $L\left(k_{(1, \infty)}\right)=1$ which is easy to see. Moreover $k_{(1, \infty)}$ is extreme, because $k_{(1, \infty)}(1, s)=0$ and thus $\mu_{g}((0, s])=0$ for every parabolic function $g$ with $g \leqq k_{(1, \infty)}$ and every $s<\infty$. Hence $(1, \infty) \in \widetilde{F}_{m}^{\prime}$. Thus (i) is proved.

If we show that the functions $\tilde{\widetilde{K}}_{0} f\left(f \in C_{c}(\widetilde{E})\right)$ separate the points of $\widetilde{F}_{m}$ then (ii) follows from [10] and (iii) is a consequence of (ii) and the preceding conclusions. Let $\tilde{y}_{0}=\left(y_{0}, t_{0}\right)$ and $\tilde{y}_{1}=\left(y_{1}, t_{1}\right) \in \widetilde{F}_{m}$ and suppose at first $t_{0}<t_{1}$. Let $f \in C_{c}(\widetilde{E}), f>0$ with support is bounded below by $\frac{t_{0}+t_{1}}{2}$ and above by $t_{1}$. Then $\tilde{\widetilde{K}}_{0} f\left(\widetilde{y}_{1}\right)>0$ and $\tilde{\widetilde{K}}_{0} f\left(\tilde{y}_{0}\right)=0$. Suppose $t_{0}=t_{1}, y_{0} \neq y_{1}$ and $\widetilde{\tilde{K}}_{0} f\left(\widetilde{y}_{0}\right)=\widetilde{\widetilde{K}}_{0} f\left(\tilde{y}_{1}\right)$ for every $f \in C_{c}(\widetilde{E})$. Then $k_{\tilde{y}_{0}}=k_{\tilde{y}_{1}}$. Let e.g. 1 be entrance. Then for $\delta<\frac{\left|y_{0}-y_{1}\right|}{2}$ we have

$$
\int_{\left|x-y_{0}\right|<\delta} k_{\tilde{y}_{i}}(\tilde{x}) m(d x)=\int_{\left|x-y_{0}\right|<\delta} p\left(t_{0}-s, x, y_{i}\right) m(d x) \underset{s \uparrow t_{0}}{\longrightarrow} 1 \quad \text { or } 0
$$

if $i=0$ or 1 respectively by the stochastical continuity of $X$. This contradicts $k_{\tilde{y}_{0}}=k_{\tilde{y}_{1}}$. If 1 is accessible by using the new pair $\left(m^{*}, p^{*}\right.$ ) (see the proof of Theorem 1) on the same way it can be shown that $k_{\tilde{y}_{0}} \neq k_{\tilde{y}_{1}}$ for $y_{0} \neq y_{1}$. Thus $\widetilde{\widetilde{K}}_{0} f\left(f \in C_{c}(\widetilde{E})\right)$ separate the points of $\widetilde{F}_{m}$. Hence the theorem is proved. 


\section{§3. Applications}

We give two applications of the preceding results. Several authors have studied the problem if every extreme parabolic function factorizes, i.e. has a representation of the form $\phi(x) \psi(t)$, and if every factorizing parabolic function is extreme (see e.g. [9], [11]).

For quasidiffusions we have the following corollary from the results above.

Corollary 1 . If 1 is entrance (accessible) the only factorizing minimal parabolic function is

$$
k_{(1, \infty)}(x)=(m(1))^{-1} \quad\left(=e^{-\lambda_{0} s} \varphi\left(x, \lambda_{0}\right)\right) .
$$

- For every $\mu>\lambda_{0}$ the factorizing parabolic function $\tilde{h}_{\mu}(\tilde{x})=e^{-\mu s} \varphi(x, \mu)$ has representation

$$
e^{-\mu s} \varphi(x, \mu)=-D_{p} \varphi(1, \lambda) \int_{s}^{\infty} e^{-\mu u} p(u-s, x, 1) d u
$$

if 1 is entrance and

$$
e^{-\mu s} \varphi(x, \mu)=\varphi(1, \mu) \int_{s}^{\infty} e^{-\mu u} D_{p} p(u-s, x, 1) d u
$$

if 1 is accessible.

Remark. The last but one formula can be proved elementary by using $\int_{0}^{\infty} e^{-\mu h} p(h, x, 1) d h=r_{\mu}(x, 1)=\varphi(x, \mu) \chi(1, \mu)=\varphi(x, \mu)\left(D_{p} \varphi(1, \mu)\right)^{-1}$ where $r_{\mu}(x, y)$ denotes the resolvent kernel density of the corresponding to $(m, p)$ semigroup $\left(S_{t}\right)$ in $C(E)$.

In [2], [8] and other papers were studied conditions under which for a given function $h(x, t)$ and a given Markov process the composition $\left(h\left(X_{t}, s+t\right)\right)_{t \geqq 0}$ is a martingale. In this connection we can formulate the following

Corollary 2. Let 1 be entrance or accessible and $f$ a parabolic function with $L(\dot{f})<\infty$ and

$$
f(\tilde{x})=\int_{0}^{\infty} k_{(1, t)}(\tilde{x}) \mu_{f}(d t) \quad(\tilde{x} \in \tilde{E}) .
$$

Then

$$
E_{x}\left(f\left(X_{t}, t+s\right) \mid \mathscr{F}_{u}\right)=f\left(X_{u}, u+s\right)-\int_{s+u}^{s+t} k_{(1, v)}\left(X_{u}, s+u\right) \mu_{f}(d v) \quad(0<u<t),
$$

where $\mathscr{F}_{u}$ denotes the $\sigma$-algebra generated by $\left\{X_{r}, r \leqq u\right\}$. In particular $\left(f\left(X_{t}, t+s\right)\right)_{t \in[a, b]}$ is a martingale with respect to every $P_{x}(x \in E)$ if and only if 
$\mu_{f}([a+s, b+s])=0$.

Proof: By the Markov property it follows

$$
\begin{aligned}
E_{x}\left(f\left(X_{t}, t+s\right) \mid \mathscr{F}_{u}\right)=E_{X_{u}}\left(f\left(X_{t-u}, t+s\right)\right)=\int_{0}^{1} f(y, t+s) p\left(t-u, X_{u}, y\right) m(d y) \\
\quad=\int_{0}^{1} \int_{t+s}^{\infty} k_{(1, v)}(y, t+s) \mu_{f}(d v) p\left(t-u, X_{u}, y\right) m(d y) \\
=\int_{t+s}^{\infty} \int_{0}^{1} k_{(1, v)}(y, t+s) p\left(t-u, X_{u}, y\right) m(d y) \mu_{f}(d v) \\
=\int_{t+s}^{\infty} k_{(1, v)}\left(X_{u}, s+u\right) \mu_{f}(d v) \\
=f\left(X_{u}, s+u\right)-\int_{s+u}^{s+t} k_{(1, v)}\left(X_{u}, s+u\right) \mu_{f}(d v) .
\end{aligned}
$$

Thereby we have used

$$
\int_{0}^{1} k_{(1, v)}(y, t) p(h, z, y) m(d y)=k_{(1, v)}(z, t-h) \quad(h<t)
$$

which follows from [5] Theorem 2 and the Chapman-Kolmogorov equation.

\section{References}

[1] Doob, J. L., Snell, J. L. and Williamson, R. E., Applications of boundary theory to sums of independent random variables, in Contributions to probability and statistics, Stanford University Press 1960.

[2] Doob, J. L., Martingales and one-dimensional diffusions, Trans. Amer. Math. Soc. 78 (1955), 168-208.

[3] Ito, K. and McKean, H. P. Jr., Diffusion processes and their sample paths, New York, Academic Press 1964.

[4] Kac, I. S. and Krein, M. G.: On the specral functicns of the string, Appendix to the Russian translation of Atkinson, Discrete and continuous boundary problcms, Moscow 1968 (in Russian).

[5] Küchler, U., Some asymptotic properties of the transition densities of quasidiffusions, Publ. RIMS, Kyoto Univ., 16 (1980), 245-268.

[6] Küchler, U. and Lunze, U., On the tail $\sigma$-field and the minimal parabolic functions for one-dimensional quasidiffusions, Z. Wahrscheinlichkeitstheorie verw. Gebiete, to appear.

[7] Kunita, H. and Watanabe, T, Markov processes and Martin boundaries I, Illinois J. Math. 9 (1965), 485-526.

[8] Lai, T. L., Space-time processes, parabolic functions and one-dimensional diffusions, Trans. Amer. Math. Soc. 175 (1973), 409-438.

[9] Lamperti, J. and Snell, J. L., Martin boundaries for certain Markov chains, J. Math. Soc. Japan, 15 (1963), 113-128.

[10] Meyer, P. A., Processus de Markov, la frontiere de Martin, Lecture Notes in Mathematics 77, Berlin 1968. 
[11] Moltschanov, S. A., Martin boundaries for the direct product of Markov processes, Sibirski Mat. Zurnal, XI (1970), 370-380 (in Russian).

[12] Robbins, H. and Siegmund, D., Boundary crossing probabilities for the Wiener process and sample sums, Ann. Math. Stat. 41 (1970), 1410-1429.

[13] Sawyer, S., A Fatou theorem for the general one-dimensional parabolic equation, Indiana University Math. Journal, 24 (1974), 451-498. 
